\title{
Assessment of Follow-Up Sonography and Clinical Improvement among Infants with Congenital Muscular Torticollis
}

\author{
H.-J. Park, S.S. Kim, S.-Y. Lee, Y.-T. Lee, K. Yoon, E.-C. Chung, M.-H. Rho, and H.-J. Kwag
}

\begin{abstract}
BACKGROUND AND PURPOSE: Infants grow rapidly, which causes the SCM to thicken physiologically. Therefore some cases of physiologically-thickened SCM can be confused with a poor response to physical therapy. There have been only a few quantitative ultrasonographic studies on the clinical outcome of rehabilitation for CMT. Our aim was to evaluate whether a new sonographic assessment method that uses the muscular thickness ratio of the SCM can help quantify the outcome of rehabilitation therapy for patients with CMT.
\end{abstract}

MATERIALS AND METHODS: We evaluated 48 patients (male/female, 17:31; mean age, 3.9 months) who were diagnosed with CMT and who underwent initial and follow-up sonography. The ratio of the thickness of the involved SCM to the thickness of the intact SCM (SCM thickness ratio) was calculated. A scoring system based on the range of motion of the neck was used to assess clinical improvement. The correlations between clinical improvement and the thickness of the involved muscle, the difference in involved muscle thickness, the SCM thickness ratio, and the difference in the SCM thickness ratio were evaluated with Spearman rank correlations.

RESULTS: Follow-up Cheng scores were higher than initial scores; this difference indicates clinical resolution (follow-up, 4.90; initial, 3.38). The SCM thickness ratio at follow-up was lower than that at the initial evaluation (follow-up, 1.29-1.34; initial, 1.65-1.77). Intra- and interobserver agreements were excellent. Most variables were moderately correlated with clinical improvement (correlation coefficients, $0.36-0.509)$. R1 showed the highest correlation with clinical improvement ( 0.481 and 0.509$)$, followed by the initial maximal thickness of the SCM (0.434 and 0.488). $\Delta \mathrm{P}$ (P1-P2) and $\Delta \mathrm{R}$ showed similar correlation coefficients with clinical improvement.

CONCLUSIONS: Measurement of the SCM thickness ratio appears to overcome the problem of a false-positive diagnosis of clinical aggravation of CMT resulting from physiologic growth. Rl and $\Delta \mathrm{R}$ are accurate objective measurements, which can be used in the management of CMT.

ABBREVIATIONS: CMT = congenital muscular torticollis; PI, 2 = initial and follow-up maximal thickness of the SCM; R1, 2 = initial and follow-up SCM thickness ratio; SCM = sternocleidomastoid muscle

$\mathbf{C}^{\circ}$ ongenital muscular torticollis is a common disorder of the musculoskeletal system in neonates and infants, with an incidence of $0.3 \%-1.9 \%$. Patients with CMT can be divided into a sternomastoid tumor group and those with tightness of sternocleidomastoid muscle but no clinical tumor as a muscular torticollis group. Postural torticollis is congenital torticollis with neither tightness nor tumor of the SCM. CMT has been used synonymously with

Received June 4, 2012; accepted after revision June 28.

From the Departments of Radiology (H.-J.P., S.-Y.L., E.-C.C., M.-H.R., H.-J.K.) and Rehabilitation Medicine (Y.-T.L., K.Y.), Sungkyunkwan University School of Medicine, Kangbuk Samsung Hospital, Seoul, Republic of Korea; and Department of Radiology (H.-J.P., S.S.K.), Kangwon National University School of Medicine, Chuncheon, Republic of Korea.

Please address correspondence to So-Yeon Lee, MD, Department of Radiology, Kangbuk Samsung Hospital, Sungkyunkwan University School of Medicine, 108

Pyung-dong, Jongno-gu, Seoul 110-746, Republic of Korea; e-mail:

radiology11@hanmail.net

http://dx.doi.org/10.3174/ajnr.A3299 congenital torticollis. ${ }^{1}$ In infants with torticollis, the head typically is tilted toward the side of the affected muscle and rotated toward the opposite side. ${ }^{2}$ On sonography, focal or diffuse enlargement of the SCM is apparent in the lower two-thirds of the muscle, and the size of the lesions ranges from 8 to $15.8 \mathrm{~mm}$ in the maximal transverse diameter. ${ }^{3}$ The echogenicity of the lesions is hyperechoic (49\%) and mixed echogenic (49\%). ${ }^{4}$

Most patients experience a reduction in the size of the SCM spontaneously or after physical therapy. ${ }^{5,6}$ However, infants grow rapidly; this growth causes the SCM to thicken physiologically, so some cases of physiologically thickened SCM can be confused with a poor response to physical therapy. To our knowledge, there have been only a few quantitative sonographic studies on the clinical outcome of rehabilitation for CMT. The purpose of this study was to evaluate whether a new sonographic assessment method that uses the muscular thickness ratio of the SCM can help quantify the outcome of rehabilitation therapy in patients with CMT. 


\section{MATERIALS AND METHODS \\ Case Selection}

This retrospective study included 48 consecutive patients (17 boys and 31 girls; mean age, $3.9 \pm 3$ months) who visited our hospital with a clinical diagnosis of CMT and who underwent initial sonography, follow-up sonography, and rehabilitation therapy between January 2009 and March 2012. The same physician from the Rehabilitation Medicine Department made the clinical diagnosis of CMT for all patients on the basis of a mass in the SCM and documentation of a deficit in passive neck rotation in association with ipsilateral SCM shortening. The exclusion criteria were postural torticollis without a mass or tightness, the presence of hematoma in the SCM, congenital anomaly of the cervical spine, spasmodic torticollis, and bilateral torticollis. Ten patients were excluded on the basis of these criteria. This study was approved by our institutional ethics review board, and the requirement for informed consent was waived because of the retrospective study design.

\section{Sonographic Evaluation}

A musculoskeletal radiologist performed all ultrasonographic examinations by using the HDI 5000 (Philips Medical Systems, Bothell, Washington) and Logiq E9 (GE Healthcare, Milwaukee, Wisconsin) imaging devices equipped with linear 6- to $15-\mathrm{MHz}$ probes. With the patient in the supine position, a small pillow was placed under the shoulder to extend the neck. This study followed our current protocol for sonographic evaluation of CMT. Specifically, a large amount of semisolid sonography transmission gel was used to minimize pressure on the lesion. Gray-scale images were obtained for both SCMs in the transverse and longitudinal planes. The largest cross-section of the lesion was captured, and the anteroposterior diameter, which represents muscular thickness, was measured. Sonograms of the involved muscle were compared with those of the uninvolved muscle, and the anteroposterior diameter of the contralateral SCM was measured (Fig. 1). The internal echotexture of the lesion was captured and classified as either homogeneous or heterogeneous. The mean interval between the 2 sonographic examinations (ie, initial and follow-up) was $4.2 \pm 3$ months. All images were retrospectively retrieved from patient files and were interpreted and measured by 2 fellowship-trained academic musculoskeletal radiologists with 10 and 12 years of experience. The ratio of the thickness of the involved SCM to the thickness of the intact SCM (ie, the SCM thickness ratio) was calculated.

\section{Clinical Correlation}

Treatment began a few days after diagnosis. Patients underwent a standard physiotherapy program that involved manual stretching
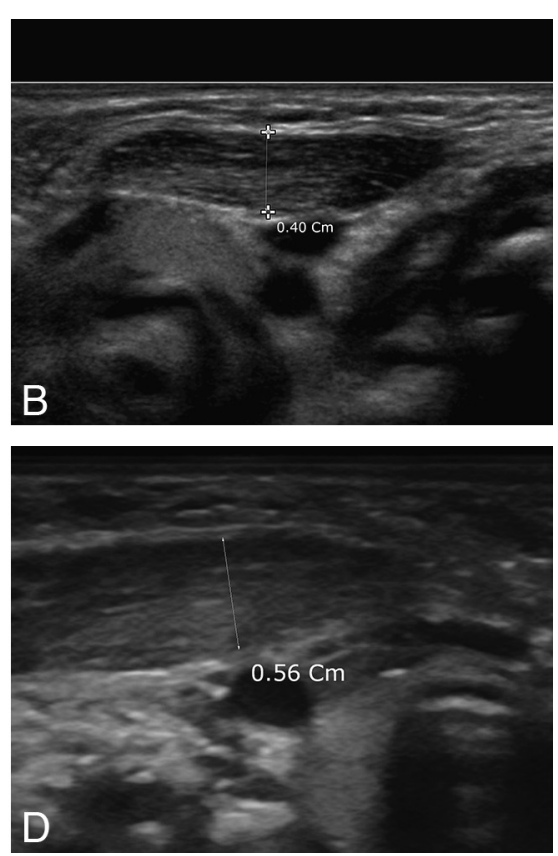

IG 1. One-month-old boy with congenital muscular torticollis on the right side. $A$, Transverse modified Cheng score was 6.

for 30 minutes 3 times per week. Manual stretching followed a standardized protocol: 3 repetitions of 15 manual stretches of the tight muscle with sustained force for 1 second and a rest period of 10 seconds between each stretch. The parents of the patients were taught to carry out a home program of active positioning in accordance with specific instructions. ${ }^{7}$ As suggested by Cheng et al, ${ }^{2}$ clinical improvement was assessed with a scoring system based on the range of motion of the neck measured with a goniometer. We adopted a modified Cheng method that considered only the numeric angles containing rotation deficits and side-flexion deficits. The degree of each angle of rotation and flexion deficit was measured and scored with a 4-point scale, respectively: 0 , poor $(>15)$; one, fair (11-15); two, good (6-10); three, excellent $(<5)$. Therefore, the modified Cheng score ranged from 0 to 6 , with higher scores indicative of better clinical results.

\section{Statistical Analysis}

The thickness of the affected SCM and the SCM thickness ratio were compared for initial and follow-up values with paired $t$ tests. Interobserver agreement between the 2 radiologists was assessed with the intraclass correlation coefficient. We used the following intraclass correlation coefficient classification system: poor reproducibility, $<0.40$; fair-to-good reproducibility, $0.40-0.75$; excellent reproducibility, $>0.75$. Wilcoxon signed-rank tests were used to evaluate differences between the initial and follow-up values (eg, difference in the SCM thickness, difference in the SCM thickness ratio). The correlation coefficients between thickness, the SCM thickness ratio, and the modified Cheng scores were calculated with categoric regression analysis and Spearman rank correlations. A weak correlation was defined as correlation coef- 
Table 1: The mean values of sternocleidomastoid muscle thickness, the SCM thickness ratio, and the modified Cheng scores ${ }^{\mathrm{a}}$

\begin{tabular}{|c|c|c|c|c|c|c|c|}
\hline & \multicolumn{2}{|c|}{$\begin{array}{c}\text { Thickness of Involved } \\
\text { Muscle }(\mathrm{cm})\end{array}$} & \multicolumn{2}{|c|}{$\begin{array}{c}\text { Thickness of Normal } \\
\text { Muscle }(\mathrm{cm})\end{array}$} & \multicolumn{2}{|c|}{$\begin{array}{l}\text { SCM Thickness } \\
\text { Ratiob }^{\text {b }}\end{array}$} & \multirow{2}{*}{$\begin{array}{l}\text { Modified Cheng } \\
\text { Score }\end{array}$} \\
\hline & Reader 1 & Reader 2 & Reader 1 & Reader 2 & Reader 1 & Reader 2 & \\
\hline Initial & $0.86( \pm 0.35)$ & $0.84( \pm 0.36)$ & $0.49( \pm 0.08)$ & $0.52( \pm 0.08)$ & $1.77( \pm 0.70)$ & $1.65( \pm 0.72)$ & $3.38( \pm 2.46)$ \\
\hline Follow-up & $0.75( \pm 0.27)$ & $0.74( \pm 0.26)$ & 0.57 (土0.09) & $0.58( \pm 0.09)$ & $1.34( \pm 0.50)$ & $1.29( \pm 0.48)$ & $4.90( \pm 1.59)$ \\
\hline
\end{tabular}

ata in parentheses are SDs.

${ }^{b}$ Ratio is the thickness of involved muscle/thickness of normal muscle.

Table 2: The intraclass correlation coefficients for the SCM thickness measurements and the SCM thickness ratio

\begin{tabular}{lcccc}
\hline & $\begin{array}{c}\text { Thickness } \\
\text { of Involved } \\
\text { Muscle }\end{array}$ & $\begin{array}{c}\text { Thickness } \\
\text { of Normal } \\
\text { Muscle }\end{array}$ & $\begin{array}{c}\text { SCM } \\
\text { Thickness } \\
\text { Ratio }\end{array}$ & $\begin{array}{c}\boldsymbol{P} \\
\text { Value }\end{array}$ \\
\hline Initial & 0.952 & 0.919 & 0.950 & $<.001$ \\
Follow-up & 0.988 & 0.935 & 0.976 & $<.001$ \\
\hline
\end{tabular}

Table 3: Correlation coefficients indicating the relationship between study variables and clinical prognosis ${ }^{a}$

\begin{tabular}{lllll}
\hline & \multicolumn{1}{c}{$\mathbf{P 1}^{\mathbf{b}}$} & \multicolumn{1}{c}{$\boldsymbol{\Delta} \mathbf{P}^{\mathbf{c}}$} & \multicolumn{1}{c}{$\mathbf{R}^{\mathbf{d}}$} & \multicolumn{1}{c}{$\boldsymbol{\Delta} \mathbf{R}^{\mathbf{e}}$} \\
\hline Reader 1 & $0.434(.002)$ & $0.384(.007)$ & $0.481(.001)$ & $0.394(.006)$ \\
Reader 2 & $0.488(<.001)$ & $0.380(.008)$ & $0.509(<.001)$ & $0.366(.011)$
\end{tabular}

a Numbers in parentheses are $P$ values. $P$ values $<.01$ were considered statistically significant.

${ }^{b} \mathrm{P} 1$ is the initial maximal thickness of the sternocleidomastoid muscle.

${ }^{c} \Delta \mathrm{P}$ is the difference in the maximal thickness of the SCM between initial and follow-up measurements.

${ }^{d} \mathrm{Rl}$ is the initial SCM thickness ratio (maximal thickness of the involved muscle/ maximal thickness of the normal muscle).

${ }^{e} \Delta R$ is the difference in the SCM thickness ratio between initial and follow-up measurements. ficients between 0.1 and 0.3 ; a moderate correlation, between 0.3 and 0.7 ; a high correlation, between 0.7 and 0.9 ; and a very high correlation, above $0.9 .^{8}$ All analyses were performed with the Statistical Package for the Social Sciences software, Version 10.1 (SPSS, Chicago, Illinois). $P$ values $\leq .05$ were considered statistically significant.

\section{RESULTS}

Table 1 depicts the mean thickness of the involved muscle and the normal muscle, the SCM thickness ratio, and the Cheng scores. Follow-up Cheng scores were higher than initial scores (mean, follow-up: $4.90 \pm 1.59$; initial: $3.38 \pm 2.46$ ), indicating clinical resolution of CMT. The SCM thickness ratio at follow-up was lower than that at initial evaluation (mean, readers 1 and 2; follow-up: $1.34 \pm 0.50,1.29 \pm 0.48$; initial: $1.77 \pm 0.70,1.65 \pm 0.72$ ). The intraclass correlation coefficient was 0.919 for thickness and 0.988 for the SCM ratio, indicating excellent interobserver agreement (Table 2). Table 3 depicts the correlation coefficients obtained from Wilcoxon signed-rank tests between the study variables and the Cheng scores; the values for most correlation coefficients were indicative of moderate correlation (0.3660.509). The initial SCM thickness ratio (R1) showed the highest correlation (0.481 and 0.509) with Cheng scores, followed by the initial maximal thickness of the SCM (P1, 0.434 and $0.488)$. The difference between initial and follow-up SCM thickness $(\Delta \mathrm{P}, \mathrm{P} 1-\mathrm{P} 2)$ and the difference between the initial and follow-up SCM thickness ratio $(\Delta \mathrm{R})$ showed a similar correlation with the Cheng scores. None of the correlation coefficients were significantly correlated. We observed 13 cases (27\%) in which the maximal thickness of the SCM was higher (ie, $\Delta \mathrm{P}<0$ ) and the SCM thickness ratio was lower (ie, $\Delta \mathrm{R}>0$ ) at follow-up than at initial evaluation (Fig 2). At follow-up, only 2 of these cases (4\%) had decreased Cheng scores, while the others had increased Cheng scores (Fig 3).

\section{DISCUSSION}

CMT typically affects first-born boys with a breech presentation or difficult obstetric history. ${ }^{3}$ The clinical and sonographic features of CMT are strongly correlated. ${ }^{6}$ In patients with CMT, sonography reveals fusiform enlargement and significant asymmetry of the SCM. Sonography also
FIG 2. Two-month-old boy with congenital muscular torticollis on the left side. A, Transverse sonogram of the left side of the neck shows the hypertrophied SCM (diameter, $0.57 \mathrm{~cm}$ ). $B$, The intact right muscle measured $0.38 \mathrm{~cm}$, and the $S C M$ thickness ratio was 1.50 . The initial modified Cheng score was 3. C, Follow-up sonography of the involved muscle shows an increased thickness of $0.68 \mathrm{~cm} 3$ months after the initial evaluation. $D$, At follow-up, the thickness of the uninvolved muscle was $0.54 \mathrm{~cm}$, the SCM thickness ratio was 1.26 , and the modified Cheng score was 6. 

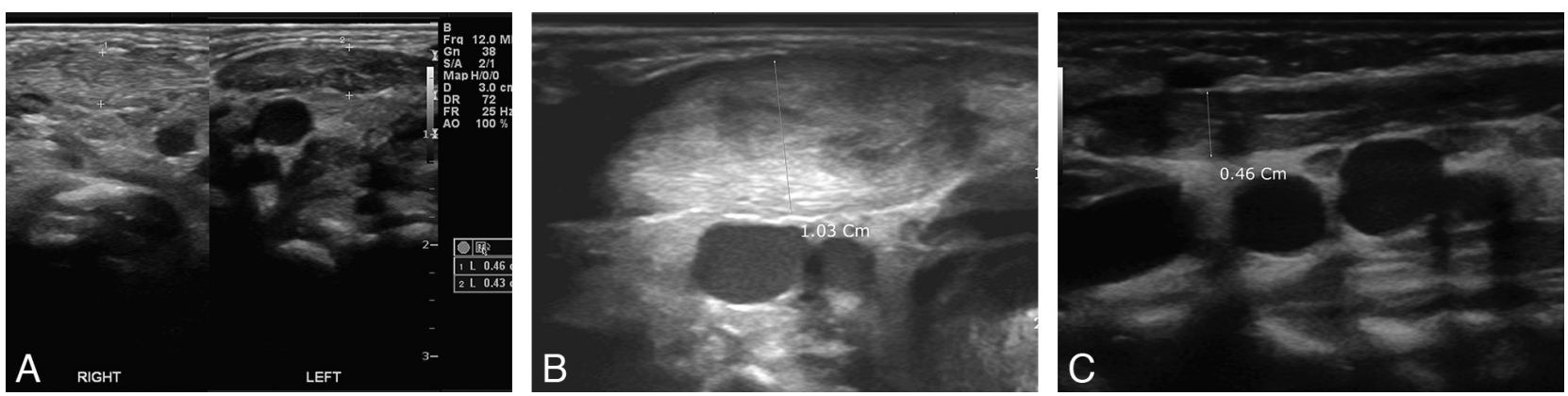

FIG 3. Five-day-old boy with congenital muscular torticollis on the right side. A, Transverse sonogram of both sides of the neck shows the hypertrophied muscle (diameter, $0.46 \mathrm{~cm}$ ) on the right side and the normal muscle on the left side (diameter, $0.43 \mathrm{~cm}$ ). The initial thickness ratio was 1.07, and the initial modified Cheng score was 0 . B. Follow-up sonography of the involved muscle shows an increased thickness of $1.03 \mathrm{~cm} 2$ months after the initial evaluation. C, At follow-up, the thickness of the uninvolved muscle was $0.46 \mathrm{~cm}$, the SCM thickness ratio was 2.24 , and the modified Cheng score was 1.

correlates well with clinical severity. ${ }^{9}$ Muscular thickening is due to the proliferation of fibrous tissue. ${ }^{10}$ Resolution of muscular thickening is a good indicator of clinical recovery and is used widely in the Department of Rehabilitation Medicine at our hospital. The echotexture of the CMT can also be a useful diagnostic tool, and the ratio of the hyperechoic lesion to the normal portion of the SCM can be used as a guideline for treatment. ${ }^{5}$ However, because hyperechoic lesions do not always show a parallel appearance along the SCM, the exact measurement of the hyperechoic lesion along the whole SCM is difficult. Furthermore, hyperechoic lesions are observed in only $49 \%$ of CMT cases. ${ }^{11}$ The maximal thickness of the involved muscle is objective and shows good interobserver agreement. In our study, the intraclass correlation coefficients for maximal thickness were extremely high (0.9190.988). This result suggests that maximal thickness of the SCM can be used as a quantitative method of evaluation. Decreased thickness generally indicates resolution of SMT, but some patients do not follow this trend. In our study, follow-up SCM thickness (P2) was higher than the initial SCM thickness (P1) in 13 patients (27\%), and the follow-up SCM thickness ratio (R2) was lower than the initial ratio (R1) in these patients. Nonetheless, all except 2 of these patients had improved Cheng scores at follow-up.

We suspect that these results can be explained by physiologic growth of the SCM. The intact contralateral SCM muscles in these patients showed increased muscle thickness (mean, $0.13 \mathrm{~cm}$ ). Therefore, an increase in the thickness of the involved muscle (ie, $\Delta \mathrm{P}<0)$ does not always indicate clinical aggravation. To compensate for changes in muscle thickness explained by physiologic growth, we suggest using the ratio of the thickness of the involved muscle to that of the normal muscle. Cheng scores were negatively correlated with both involved SCM thickness and the SCM thickness ratio. The initial SCM thickness ratio was more strongly correlated with the Cheng scores at follow-up than was the initial SCM thickness or the change in the initial SCM thickness. The change in the SCM ratio and the change in the involved SCM thickness showed a similar correlation with the Cheng score. Although the correlation coefficient for P1 was slightly higher than that for $\Delta \mathrm{P}$ and though the correlation coefficient for $\mathrm{R} 1$ was slightly higher than that for $\Delta \mathrm{R}$, there were no significant differences between any of the correlation coefficients.

We used modified Cheng scores to evaluate clinical improve- ment in patients with CMT. The original Cheng score is composed of 2 objective measurements of neck motion and 3 subjective criteria (craniofacial asymmetry, palpable residual band, and subjective head tilting). ${ }^{2}$ We used the 2 objective measurements only to produce a modified Cheng score that ranged from 0 to 6 . We focused on whether $\mathrm{R} 1$ and $\Delta \mathrm{R}$ might show a close correlation with clinical improvement, and we compared these correlations with those of $\mathrm{P} 1$ and $\Delta \mathrm{P}$. The correlation coefficient for $\mathrm{P} 1$ was higher than that for $\Delta \mathrm{P}$, and the correlation coefficient for $\mathrm{R} 1$ was higher than that for $\Delta \mathrm{R}$. The correlation coefficient for R1 was slightly higher than that for $\mathrm{P} 1$, but the correlation coefficients for the $\Delta \mathrm{P}$ and the $\Delta \mathrm{R}$ were similar. Thus, we found that $\mathrm{R} 1$ and $\mathrm{P} 1$ reflect clinical improvement in CMT, while the $\Delta \mathrm{P}$ and $\Delta \mathrm{R}$ may also indicate clinical improvement. The $\Delta \mathrm{R}$ may be particularly helpful in the interpretation of the mismatch between clinical improvement and sonographic findings among patients who have higher $\mathrm{P} 2$ than $\mathrm{P} 1$, as occurred among 13 patients in our study.

Our study has some limitations. First, we were unable to evaluate the correlation between increased echogenicity and clinical improvement. Quantitative analysis of increased echogenicity was difficult, and some cases did not show increased echoes in the involved SCM. Another limitation was the retrospective study design, which made it difficult to perform a completely blinded study.

\section{CONCLUSIONS}

$\mathrm{P} 1, \mathrm{R} 1, \Delta \mathrm{P}$, and $\Delta \mathrm{R}$ were moderately correlated with clinical improvement. The SCM thickness ratio overcomes the error of a false-positive diagnosis of clinical aggravation of the CMT resulting from physiologic growth. $\mathrm{R} 1$ and $\Delta \mathrm{R}$ are accurate objective measurements that can be used in the management of CMT.

\section{REFERENCES}

1. Cheng JC, Metreweli C, Chen TM, et al. Correlation of ultrasonographic imaging of congenital muscular torticollis with clinical assessment in infants. Ultrasound Med Biol 2000;26:1237-41

2. Cheng JC, Tang SP, Chen TM, et al. The clinical presentation and outcome of treatment of congenital muscular torticollis in infants: a study of 1,086 cases. J Pediatr Surg 2000;35:1091-96

3. Dudkiewicz I, Ganel A, Blankstein A. Congenital muscular torticollis in infants: ultrasound-assisted diagnosis and evaluation. J Pediatr Orthop 2005;25:812-14

4. Chan YL, Cheng JC, Metreweli C. Ultrasonography of congenital muscular torticollis. Pediatr Radiol 1992;22:356-60

AJNR Am J Neuroradiol 34:890-94 Apr 2013 www.ajnr.org 
5. Lin JN, Chou ML. Ultrasonographic study of the sternocleidomastoid muscle in the management of congenital muscular torticollis. J Pediatr Surg 1997;32:1648-51

6. Tang SF, Hsu KH, Wong AM, et al. Longitudinal follow up study of ultrasonography in congenital muscular torticollis. Clin Orthop Relat Res 2002;403:179-85

7. Lee YT, Yoon KJ, Kim YB, et al. Clinical features and outcome of physiotherapy in early presenting congenital muscular torticollis with severe fibrosis on ultrasonography: a prospective study. $\mathrm{J} \mathrm{Pe}$ diatr Surg 2011;46:1526-31
8. Cohen J. Statistical Power Analysis for the Behavioral Sciences. 2nd ed. Hillsdale, New Jersey: Lawrence Erlbaum Associates; 1988

9. Tatli B, Aydinli N, Cahskan M, et al. Congenital muscular torticollis: evaluation and classification. Pediatr Neurol 2006;34:41-44

10. Hsu TC, Wang CL, Wong MK, et al. Correlation of clinical and ultrasonographic features in congenital muscular torticollis. Arch Phys Med Rehabil 1999;80:637-41

11. Ablin DS, Jain K, Howell L, et al. Ultrasound and MR imaging of fibromatosis colli (sternomastoid tumor of infancy). Pediatr Radiol 1998;28:230-33 